\title{
Early slip activity and fatigue crack initiation of a near alpha titanium alloy
}

\author{
Conghui Liu*, Rhys Thomas, João Quinta da Fonseca, Michael Preuss \\ Materials Performance Centre, School of Materials, the University of Manchester, Manchester, M13 9PL, UK. \\ * Corresponding author. E-mail address: conghui.liu@postgrad.manchester.ac.uk
}

\begin{abstract}
For titanium alloys, crack initiation as a result of plastic strain accumulation has been shown to govern fatigue life under the high cycle fatigue regime. In this study, the early plastic slip activity and fatigue crack initiation was studied using a cyclic four point bending test at $10 \mathrm{~Hz}$ with a load ratio of 0.1 , up to $90 \%$ of the proof stress. The plastic slip in the high stress area was monitored by interrupting the test and performing optical microscopy. Following fatigue crack initiation, scanning electron microscopy (SEM) combined with electron backscatter diffraction (EBSD) was used to identify the slip and crack initiation mode in a $600 \mathrm{x} 600 \mu \mathrm{m}^{2}$ area. Using slip trace analysis, it was shown that primary alpha grains offered dominant plastic deformation with basal slip activation. Cracking along basal planes was determined to be the dominant damage mode.
\end{abstract}

Keywords: TIMETAL ${ }^{\circledR} 834$; Fatigue; Slip; Crack initiation

\section{Introduction}

TIMETAL ${ }^{\circledR} 834$ is a near- $\alpha$ titanium alloy developed for high temperature (up to $600{ }^{\circ} \mathrm{C}$ ) service parts in gas turbine engines [1]. Its bimodal microstructure consisting of equiaxed primary alpha $\left(\alpha_{\mathrm{p}}\right)$ grains located at the triple-point of the $\beta$ grain boundaries and secondary alpha $\left(\alpha_{\mathrm{s}}\right)$ colonies is produced by deformation in the $\alpha+\beta$ phase region with subsequent recrystallization and ageing. By changing deformation conditions and cooling rates during processing, critical microstructural parameters including volume fraction and size of primary alpha grains, $\beta$ grain size and secondary alpha colony size can be tailored to achieve certain material properties. It has been reported that TIMETAL $^{\circledR} 834$, with a bimodal microstructure and around $15 \%$ primary alpha grains exhibits well-balanced creep-fatigue properties [2].

During service, high cycle fatigue (HCF) has been found to be the largest single cause of component failure [3]. Although previous work has shown that fatigue damage is the result of the accumulation of localized plastic deformation during cyclic loading, which is directly related to the microstructural heterogeneities among constituent grains [35], the relevance of slip activation during fatigue loading is still not clear. Some research suggests prismatic slip dominates [6,7] while other work proposes that basal slip is more prominent, depending on the composition of the alloy [8]. For instance, it has been claimed that prismatic slip is favoured in pure Ti, whilst basal is increasingly favoured with the addition of $\alpha$-stabilisers [9]. Numerous experimental studies have reported that the mechanism of fatigue crack initiation is correlated with the slip bands across primary alpha grains or secondary alpha colonies in bimodal microstructures $[7,8,10,11]$. Therefore, to have a better understanding of the plastic deformation and fatigue crack initiation in TIMETAL ${ }^{\circledR} 834$, a statistical analysis of slip system activity and its link to crack initiation is required.

In the present paper, we focus on the micromechanical plastic deformation and mechanisms of fatigue crack initiation in bimodal TIMETAL ${ }^{\circledR} 834$ alloy. Early plastic deformation was studied by interrupted fatigue tests under four point bending. The fatigue crack initiation was monitored by optical and scanning electron microscopy (SEM) from the specimen surface where stress is highly concentrated. The studied areas covered hundreds of grains and slip trace analysis was used to identify active slip systems in the same area by SEM combined with grain orientation data by Electron Backscatter Diffraction (EBSD).

\section{Materials and experimental procedure}

The material utilised for this study was a TIMETAL ${ }^{\circledR} 834$ forged bar (with a diameter of $250 \mathrm{~mm}$ ) provided by TIMET UK Ltd. In order to create a bimodal microstructure, a two-step heat treatment was undertaken consisting of a solution heat treatment at $1015{ }^{\circ} \mathrm{C}$ for 2 hours followed by oil quenching to acquire a microstructure with equiaxed primary alpha $\left(\alpha_{\mathrm{p}}\right)$ grains in the lamellar secondary alpha $\left(\alpha_{\mathrm{s}}\right)$ colonies. Subsequent ageing at $700^{\circ} \mathrm{C}$ for two hours was employed to release the residual stresses induced in the previous step. It is also well established that such ageing heat treatment promotes $\mathrm{a}_{2}$ formation [12]. All heat treatments were conducted in a tube furnace under an argon atmosphere. The final microstructure is presented in Figure 1. (b), which reveals that the volume fraction of primary alpha is at approximately $25 \%$ with $75 \%$ remaining as secondary alpha colonies and a very small fraction $\mathrm{b}$ ligaments retained from the $\mathrm{b}$ grains present during the solution heat treatment.

The forged bar was machined into $25 \times 3 \times 4 \mathrm{~mm}^{3}$ specimens by wire electric discharge machining for testing. For EBSD and SEM studies, samples were prepared by grinding with $\mathrm{SiC}$ paper from $\# 400$ to $\# 4000$ and finally OPS polishing for 20 minutes in a suspension consisting of $80 \% 0.05 \mu \mathrm{m}$ OPS and $20 \%$ hydrogen peroxide. After preparation, microhardness indents were placed to denote the area of interest for microscopy.

Four point bending fatigue test was carried out at room temperature on un-notched samples. The distance of inner rollers was set to $4 \mathrm{~mm}$ apart and the loading rollers to $15 \mathrm{~mm}$ apart, which supports a $3 \times 4 \mathrm{~mm}^{2}$ maximum stress area on the tensile surface. The fatigue test was performed in a load control mode with a sine waveform signal at a frequency $f=10 \mathrm{~Hz}$, a ratio $R_{\sigma}=\sigma_{\min } / \sigma_{\max }=0.1$ and $\sigma_{\max }=840 \mathrm{MPa}\left(\sigma_{0.2}=927 \mathrm{MPa}\right)$ using a Bose $3 \mathrm{kN}$ testing machine. The stress along the tensile surface was calculated using the following equation [13]:

$$
\sigma=3 F\left(L-L_{i}\right) / 2 b d^{2}
$$

(C) The Authors, published by EDP Sciences. This is an open access article distributed under the terms of the Creative Commons Attribution License 4.0 (http://creativecommons.org/licenses/by/4.0/). 
Where $L$ is the distance between loading rollers, $L_{i}$ is the distance between the inner rollers, $b$ is the width of the sample, $d$ is the depth, $F$ is the load and $\sigma$ is the stress on the tensile surface. The advantage of four-point bending over three-point bending is that more crack initiation sites would be captured in the uniform stress distribution area.

The early plastic deformation in a bimodal TIMETAL ${ }^{\circledR} 834$ specimen was characterized by interrupted fatigue testing. Optical microscopy provides a quick approach to monitoring the development of plastic deformation and crack initiation. Slip trace analysis was performed in an area of $600 \times 600 \mu \mathrm{m}^{2}$ located at the centre of the high stress area as shown in the Figure 1 (a) comparing slip traces recorded by SEM with theoretical slip trace angles determined using grain orientation obtained by EBSD. EBSD scans were performed at an operating voltage of $20 \mathrm{kV}$ and a step size of $1 \mu \mathrm{m}$ using a TESCAN field emission gun SEM. The SEM images were acquired in Back-Scattered Electron (BSE) mode to have high contrast between primary alpha grains and secondary alpha colonies. A $\pm 5^{\circ}$ criterion was used to compare the theoretical and observed slip traces. Pyramidal slip systems were not considered in this study due to the very low activation frequency in the low stress range and only basal and prismatic slip system were studied. Among the three slip directions in the basal plane, the one with the highest Schmid factor was assumed to be the active one.

(a)

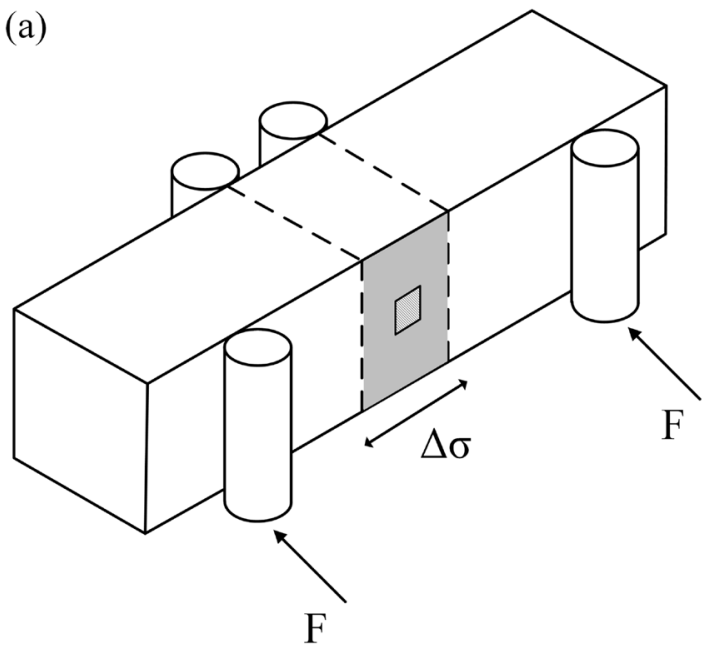

(b)

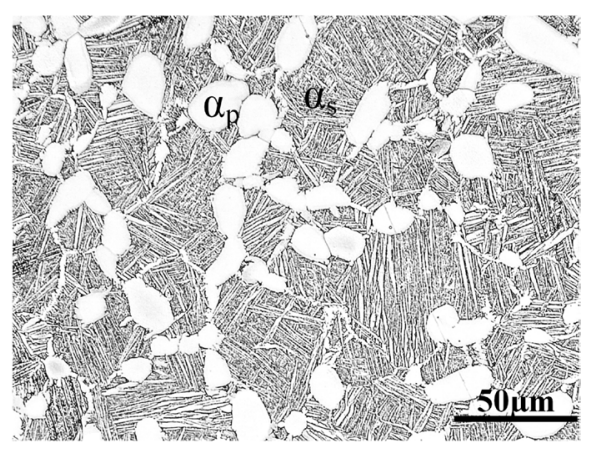

Figure 1. (a) Schematic of the four-point bending fatigue test; (b) optical micrograph of the bimodal microstructure of TIMETAL ${ }^{\circledR} 834$.

\section{Results and Discussion}

3.1 Development of plastic deformation during interrupted fatigue test

The development of plastic deformation on the sample surface was tracked in an area measuring $600 \times 600 \mu \mathrm{m}^{2}$. This area was selected from the region of high uniform stress located between the two inner rollers. As presented in Figure 2, there are 52 primary alpha grains with slip activation after 1000 cycles, 53 primary alpha grains with slip activation after 20,000 cycles and 56 primary alpha grains with slip activation after 60,000 cycles. With the cycling number increasing to 100,000 , the active primary alpha grains is still 56 but monitored with crack initiation. Besides, SEM observation reveals that $95 \%$ slip activity is restricted to primary alpha grains which accommodate the dominant plastic deformation. Therefore, the slip system activity in the secondary alpha colonies is not discussed in the present work. Slip trace analysis was carried out after 60000 cycles with 56 primary alpha grains among 246 primary alpha grains in total exhibiting slip activation. 

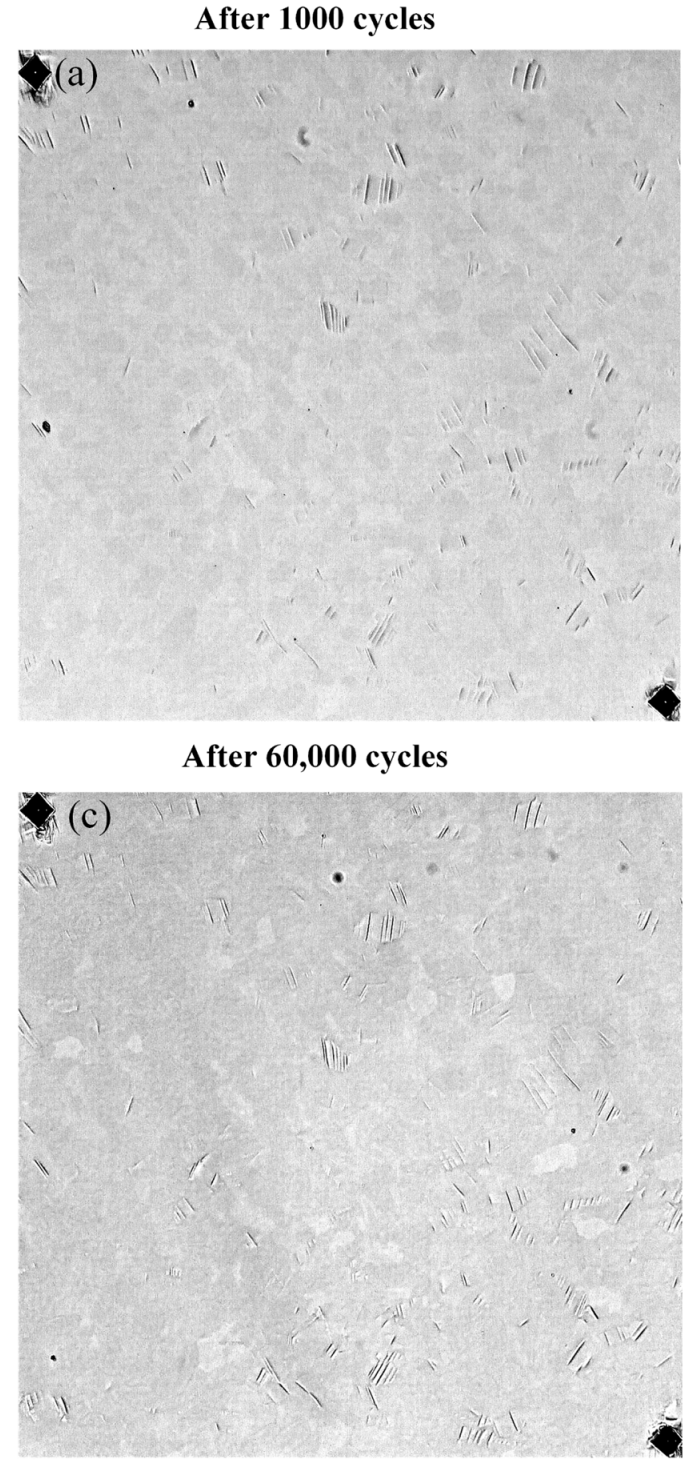

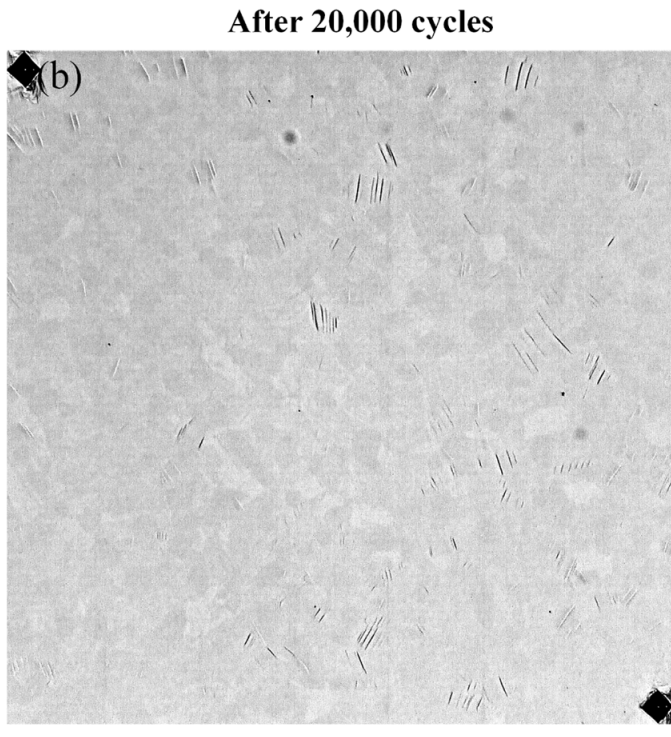

After 100,000 cycles

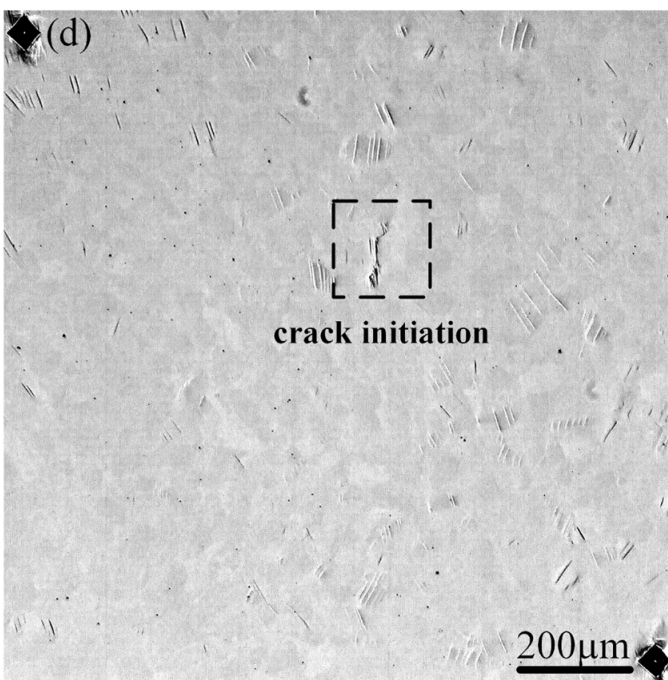

Figure 2. Optical images of the development of slip activities in different cycles: (a) $52 \alpha_{p}$ grains with slip activation after 1000 cycles; (b) $53 \alpha_{p}$ grains with slip activation after 20000 cycles; (c) $56 \alpha_{p}$ grains with slip activation after 20000 cycles; (d) $56 \alpha_{p}$ grains with slip activation after 100000 cycles.

3.2 Distribution of slip activation related to Schmid factor

Figure 3 compares the histogram of Schmid factor distribution predicted by EBSD with the Schmid factor distribution of the active slip system based on slip trace angles determined from SEM by slip trace analysis. Firstly, to predict the Schmid factor distribution for the two easy active slip systems in primary alpha grains, those distributions were calculated based on the maps recorded by EBSD. It should be noted that this analysis did not include any areas of secondary alpha and the total number of primary alpha grain was 246. Figure 3 (a) shows the prismatic and basal Schmid factor distributions demonstrating that there is a slightly higher fraction of grains well aligned for basal than prismatic slip. It is also noticeable that while basal slip is distributed over a relatively wide Schmid factor range, potential prismatic slip is constrained to only high ( $>0.35$ ) Schmid factors. For example, for the highest Schmid factor binning range $(0.45-0.5)$, about $27 \%$ of primary alpha grains are associated with potential basal slip whereas only $16 \%$ of primary alpha grains are associated with potential prismatic slip. Based on those distributions one might expect to see slightly more basal than prismatic slip traces in the studied region. Figure 3 (b) displays the outcome of the slip trace analysis after 60,000 fatigue cycles plotting the frequency of prismatic and basal slip traces as a function of Schmid factor. The analysis revealed that only 56 of the 246 primary $\alpha$ grains has developed any slip traces and only one grain exhibited prismatic slip, the frequency of which is much lower than predicted in Figure 3 (a). 
(a)

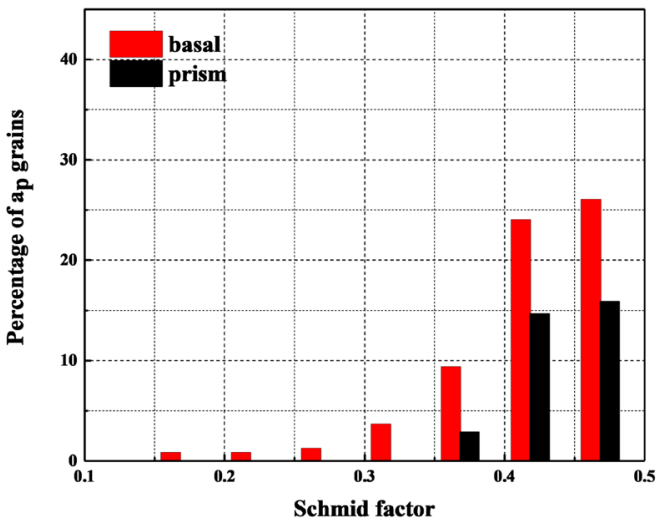

(b)

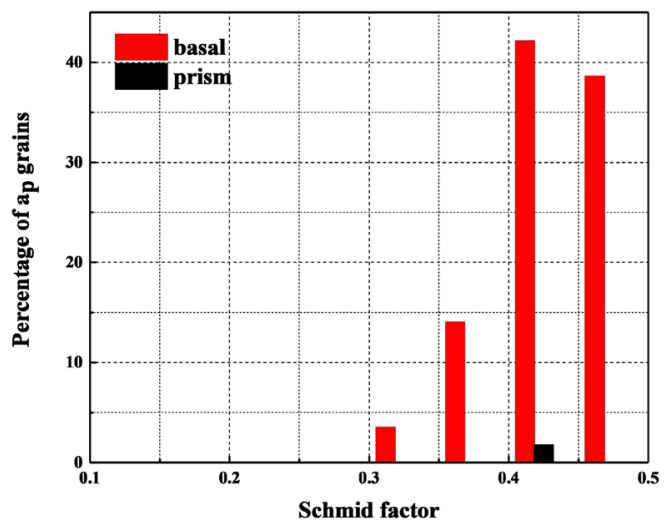

Figure 3. (a) Distribution of likely slip systems activation in $\alpha_{p}$ grains according to Schmid factor from the studied area; (b) distribution of observed slip systems after 60000 cycles as a function of Schmid factor.

The dominance of basal slip systems has been clearly shown. In order to discuss whether or not local crystallographic orientation will influence the slip activation, the orientation of each primary alpha grain in the analysed area with respect to the tensile axis was projected on an inverse pole figure (IPF, Figure 5). It is clear that due to the lack of significant texture, the grains are distributed homogeneously on the unit triangle. The basal and prismatic slips are also indicated on the IPF with red dots corresponding to active basal slip and blue dots to prismatic slip. Prismatic slip was observed as the preferential deformation in $\alpha$-titanium single crystals with the lower critical resolved shear stress (CRSS). The competition of different slip activation will be influenced by chemical composition in Ti alloys. With an addition of aluminium and interstitial elements increase, CRSS value of prismatic slip system will increase. It was indicated that basal slip becomes equally favoured to prismatic slip when the aluminium content exceeds to $6.6 \mathrm{wt} \%$ [14]. The elastic anisotropy of the hexagonal close-packed primary alpha phase, depending on the angle between the c - axis and tensile stress direction [2], needs to be considered too in the studied loading stress area. It reaches the maximum when the tensile stress direction is parallel to the $\mathrm{c}$ - axis, which means higher level of stress concentration near the [0001] pole of the IPF. Therefore, basal slip is more likely to activate compared to prismatic slip when the Schmid factor is similar. This consideration might explain the apparently low frequency of prismatic slip activation.

3.3 Fatigue crack initiation related to local grain orientation

In this study, crack initiation is defined as a crack with length comparable to the scale of the smallest microstructure features [15]. All cracks were observed to initiate across a primary alpha grain and EBSD data shows that all the cracks in the fractured grains occur along the basal plane. An example of this is demonstrated in Figure 4. For example, the crack in grain 1 occurs along a basal plane with Schmid factor 0.429 , which is moderate $(<0.45)$ among all active basal grains. This kind of fatigue damage related to slip planes across $\alpha_{p}$ grains in bimodal microstructure of Ti alloys is in agreement with previous studies [7, 8, 16]. Based on Stroh's model, it was proposed that fatigue crack initiation along the basal planes is due to the restricted slip activities on this plane. So the slip of the soft grains could transfer shear stress into the neighbouring hard grains across the grain boundaries and thereby introduce a tensile stress in the hard grains and cause a slip band to form, which then results in the cleavage of basal plane [17, 18]. However, in the materials studied here, slip occurs primarily in these hard orientaed grains, which deform by basal slip. The fractured grain is located around the [0001] pole shown (Figure 5), where tensile stress component is high. The basal cracks lies on the basal planes with a relatively high resolved shear stress combined with high resolved tensile stress.

(a)

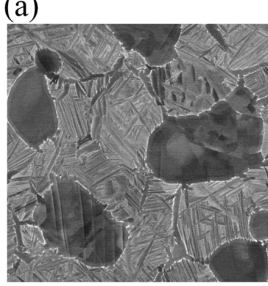

(b)

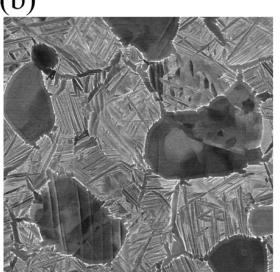

(c)

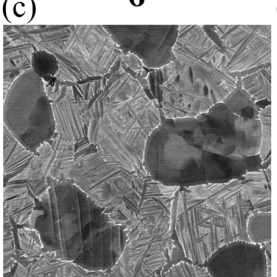

(d)
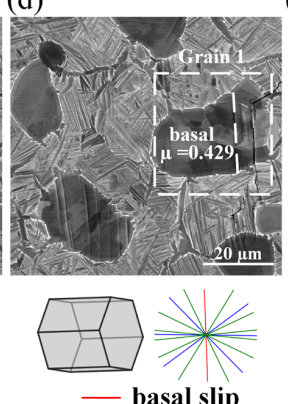

_ basal slip

— prismatic slip

— pyramidal slip (e)

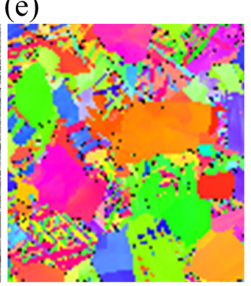

0001 $-12-10$

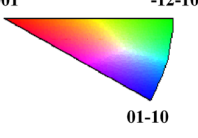

Figure 4. An example of basal crack in grain1: BSE image of the microstructure (a) after 6000 cycles; (b) after 20,000 cycles; (c) after 100,000 cycles and (d) EBSD image in loading direction of tensile stress. 


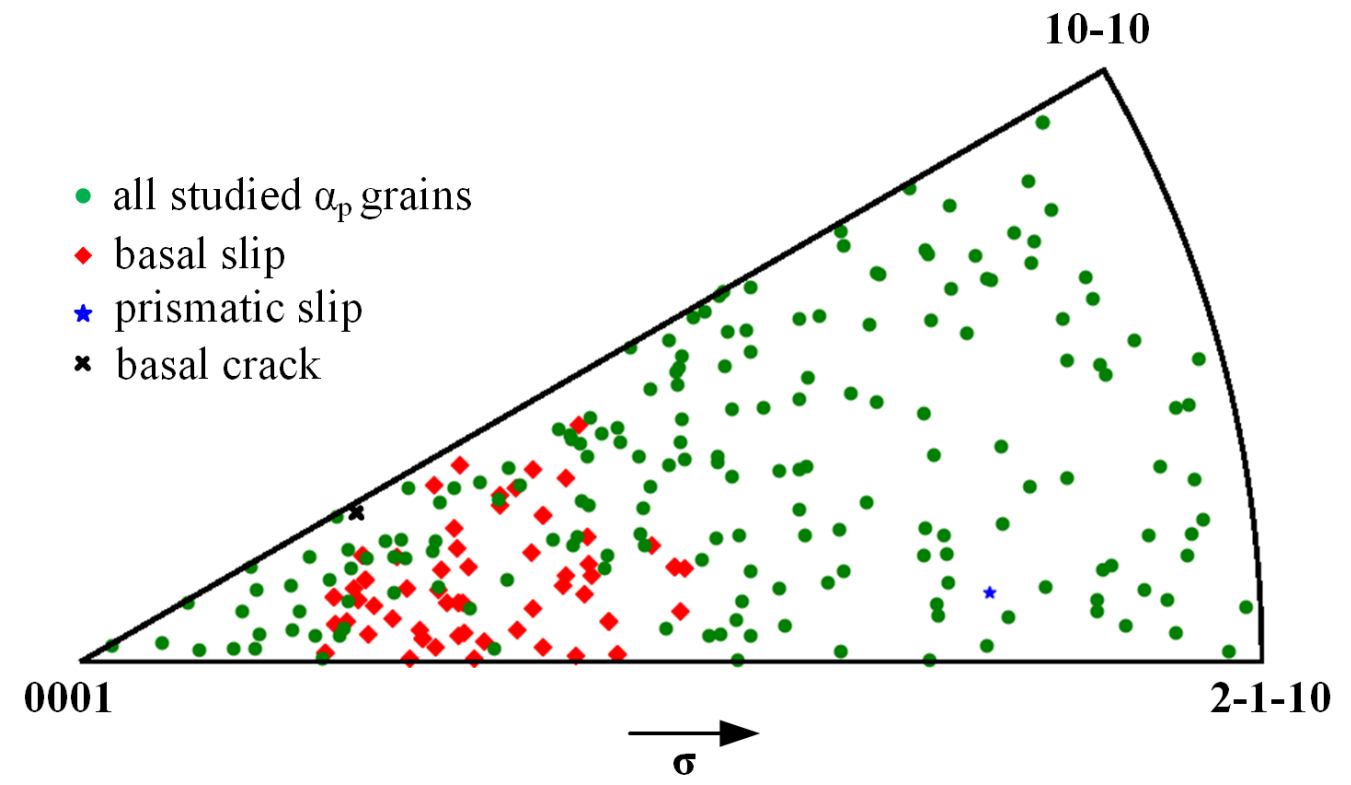

Figure 5. Crystallographic orientation of $\alpha_{p}$ grains which shows basal slip (red), prismatic slip (blue) and basal crack (black triangle), where crystal orientations are relative to the tensile axis.

\section{Conclusions}

The early plastic deformation and fatigue crack initiation was studied in a bimodal TIMETAL ${ }^{\circledR} 834$ alloy using a combination of optical microscopy, SEM and EBSD. The results can be summarized as follows:

(1) Based on a statistical analysis on 246 grains, primary alpha grains offered dominant plastic deformation compared to secondary alpha colonies.

(2) Basal slip was found to be by far the dominant active slip system under stress load controlled fatigue test below the $0.2 \%$ proof stress despite the local texture providing also plenty of grains well aligned for prismatic slip.

(3) Fatigue crack initiation occurred in the primary alpha grains along the basal planes. The grain displayed a moderate Schmid factor for basal slip ( 0.43$)$ and an angle of $29^{\circ}$ between the c-axis and the tensile loading direction.

\section{Acknowledgements}

C. Liu would like to acknowledge funding by President's Doctoral Scholar award from the University of Manchester. The author would also like to thank the Mechanics of Microstructures group at the University of Manchester to providing the data analyse code. J. Quinta da Fonseca acknowledges EPSRC funding for the LightForm programme grant (EP/R001715/1). M. Preuss also acknowledges EPSRC funding for the HEXMAT programme grant (EP/K034332/1).

\section{References}

[1] R.R. Boyer, An overview on the use of titanium in the aerospace industry, Mat Sci Eng a-Struct 213(1-2) (1996) 103-114.

[2] G. Lütjering, J.C. Williams, Titanium, Springer Science \& Business Media2007.

[3] M.D. Sangid, The physics of fatigue crack initiation, International Journal of Fatigue 57 (2013) 58-72.

[4] H. Mughrabi, Cyclic slip irreversibility and fatigue life: A microstructure-based analysis, Acta Materialia 61(4) (2013) 1197-1203.

[5] S. Suresh, R.O. Ritchie, Propagation of short fatigue cracks, International Materials Reviews 29(1) (1984) 445-475.

[6] S. Birosca, J.Y. Buffiere, M. Karadge, M. Preuss, 3-D observations of short fatigue crack interaction with la2mellar and duplex microstructures in a two-phase titanium alloy, Acta Materialia 59(4) (2011) 1510-1522.

[7] F. Bridier, P. Villechaise, J. Mendez, Slip and fatigue crack formation processes in an $\alpha / \beta$ titanium alloy in relation to crystallographic texture on different scales, Acta Materialia 56(15) (2008) 3951-3962.

[8] G.J. Baxter, Fatigue damage accumulation in titanium alloy IMI 834, University of Sheffield, 1994

[9] M. Legros, A. Corn, D. Caillard, Prismatic and basal slip in Ti3Al I. Frictional forces on dislocations, Philosophical Magazine A 73(1) (1996) 61-80. 
[10] C. Huang, Y. Zhao, S. Xin, W. Zhou, Q. Li, W. Zeng, C. Tan, High cycle fatigue behavior of Ti-5Al-5Mo-5V-3Cr-1Zr titanium alloy with bimodal microstructure, Journal of Alloys and Compounds 695 (2017) 1966-1975.

[11] C.J. Szczepanski, S.K. Jha, J.M. Larsen, J.W. Jones, Microstructural Influences on Very-High-Cycle Fatigue-Crack Initiation in Ti-6246, Metallurgical and Materials Transactions A 39(12) (2008) 2841-2851.

[12] G. Lütjering, Influence of processing on microstructure and mechanical properties of $(\alpha+\beta)$ titanium alloys, Materials Science and Engineering: A 243(1-2) (1998) 3245 .

[13] W.D. Callister, D.G. Rethwisch, Materials science and engineering, John Wiley \& Sons NY2011.

[14] J. Williams, R. Baggerly, N. Paton, Deformation behavior of HCP Ti-Al alloy single crystals, Metallurgical and Materials Transactions A 33(3) (2002) 837-850.

[15] J. Hall, Fatigue crack initiation in alpha-beta titanium alloys, International journal of fatigue 19(93) (1997) 23-37.

[16] K.S. Chan, Y.D. Lee, Effects of Deformation-Induced Constraint on High-Cycle Fatigue in Ti Alloys with a Duplex Microstructure, Metallurgical and Materials Transactions A 39(7) (2008) 1665-1675.

[17] M. Bache, A review of dwell sensitive fatigue in titanium alloys: the role of microstructure, texture and operating conditions, International Journal of Fatigue 25(9-11) (2003) 1079-1087.

[18] M. Bache, M. Cope, H. Davies, W. Evans, G. Harrison, Dwell sensitive fatigue in a near alpha titanium alloy at ambient temperature, International journal of fatigue 19(93) (1997) 83-88. 